Check for updates

Cite this: Chem. Commun., 2017, 53,12580

Received 22nd September 2017, Accepted 10th October 2017

DOI: $10.1039 / \mathrm{c} 7 \mathrm{cc} 07416 f$

rsc.li/chemcomm

\section{Azulene-boronate esters: colorimetric indicators for fluoride in drinking water $\dagger$}

\author{
Carlos M. López-Alled, (D) a Adrian Sanchez-Fernandez, (D) ${ }^{\text {bc }}$ Karen J. Edler, (D) ab \\ Adam C. Sedgwick, (ID b Steven D. Bull, iD ab Claire L. McMullin, iD b \\ Gabriele Kociok-Köhn, (iD) ${ }^{d}$ Tony D. James, (D) *ab Jannis Wenk (D) *ae and \\ Simon E. Lewis iD *ab
}

Low cost and in situ fluoride detection by non-experts is important for the determination of drinking water safety in developing countries. Colour reagents can provide results quickly without expensive equipment, but colorimetric fluoride indicators are often nonspecific, complex to use or do not work in water. Here we show that azulene-boronate indicators respond selectively to fluoride at concentrations relevant to the WHO limit of $1.5 \mathrm{mg} \mathrm{L}^{-1}$.

Fluoride occurs at trace levels in all natural waters. ${ }^{1-3}$ The highest fluctuations of natural fluoride concentrations in the aquatic environment can be found in groundwater ranging from $0.1 \mathrm{mg} \mathrm{L}^{-1}$ to well above $30 \mathrm{mg} \mathrm{L}^{-1}, 4$ depending on the local geology. Areas that are particularly prone to high groundwater fluoride concentration include parts of Africa, China, the Middle East and Southern Asia. ${ }^{5}$

The window between beneficial and harmful effects upon fluoride uptake by humans is small. Low levels of fluoride intake reduce the risk of dental caries. ${ }^{6}$ Higher levels of fluoride lead to adverse health effects, causing dental and skeletal fluorosis and kidney failure. ${ }^{7}$ The overall recommended fluoride uptake for humans is $0.05 \mathrm{mg} \mathrm{kg}^{-1}$ body weight per day with a tolerable upper intake of $10 \mathrm{mg}$ per day. ${ }^{8}$ Such an uptake can easily be reached in regions where ground water that is naturally high in fluoride is the main source of drinking water. It is estimated that $>200$ million people globally are dependent on drinking water with a fluoride level exceeding the World Health

\footnotetext{
${ }^{a}$ Centre for Sustainable Chemical Technologies, University of Bath, Bath, BA2 7AY, UK.E-mail:T.D.James@bath.ac.uk,J.H.Wenk@bath.ac.uk, S.E.Lewis@bath.ac.uk

${ }^{b}$ Department of Chemistry, University of Bath, Bath, BA2 7AY, UK

${ }^{c}$ European Spallation Source, Lund, 221 00, Sweden

${ }^{d}$ Chemical Characterization and Analysis Facility, University of Bath, Bath, $B A 27 A Y, U K$

${ }^{e}$ Department of Chemical Engineering \& Water Innovation \& Research Centre: WIRC @ Bath, University of Bath, Bath, BA2 7AY, UK

$\dagger$ Electronic supplementary information (ESI) available: UV/Vis spectra. Titration with fluoride. Computational methodology, data and geometries. Synthetic details. Details of SAXS studies. CCDC 1568176 (Az-1-Bpin). For ESI and crystallographic data in CIF or other electronic format see DOI: 10.1039/c7cc07416f
}

Organization (WHO) guideline of $1.5 \mathrm{mg} \mathrm{\textrm {L } ^ { - 1 }}$.,10 The EPA Maximum Contaminant Level for fluoride in drinking water is $4.0 \mathrm{mg} \mathrm{L}^{-1}{ }^{11}$

A variety of fluoride detection methods have been developed. ${ }^{12}$ Standard lab methods to measure fluoride in water include ion-selective electrodes, colorimetric, distillation and ionchromatography methods. ${ }^{13}$ Commercially available portable tests (e.g. Hach, Hannah, Merck) for field measurements of fluoride employ the traditional colorimetric zirconium complex based SPADNS method ${ }^{14}$ or ion-selective electrodes (e.g. Extech, Mettler-Toledo, Thermoscientific).

Recent reviews summarise the developments of fluorescent or colorimetric fluoride chemosensors and chemodosimeters in the past few years. ${ }^{15-18}$ Several popular receptor design strategies have emerged for fluoride sensing at the molecular level. The strong affinity of fluoride for silicon has led to many reports on indicators with a receptor motif that comprises a $\mathrm{Si}-\mathrm{O}$ or $\mathrm{Si}-\mathrm{C}$ bond. Fluoride mediated modulation of hydrogen bonding $^{19}$ and fluoride mediated ligand displacement have also been employed. ${ }^{20}$ Fluorescent lanthanide complexes have been reported that have the advantage of working in pure water. ${ }^{21,22}$ One strategy has been to use receptor motifs comprising a boron-containing functional group which can reversibly bind fluoride. ${ }^{16}$ We have previously reported fluoride sensors that employ boron. ${ }^{23}$

We have developed colorimetric fluoride indicators that consist of a boronate ester receptor motif conjugated to an azulene reporter motif. Azulene is a bicyclic aromatic hydrocarbon, notable for its unusually high polarity and its deep blue colour $;{ }^{24}$ we have previously described methodology for azulene derivatization..$^{25}$ The absorption spectra for azulene derivatives are very sensitive to the electronic nature of the substituents on the azulene ring. ${ }^{26}$ We sought to exploit this property of azulene to develop new colorimetric fluoride indicators. Many prior reports of azulene-based colorimetric indicators for various analytes exist, including phosphate,${ }^{27-30}$ acetate,${ }^{30}$ cyanide, ${ }^{30}$ hydrogen peroxide, ${ }^{31}$ silver, ${ }^{32}$ mercury and other heavy metals. ${ }^{33-35}$ (Some of these indicators are not wholly selective and also 
exhibit a colorimetric response to fluoride $\mathrm{e}^{27-30}$ ). Of closest relevance to our present work is a report from Eichen et al. ${ }^{36}$ which describes a fluorescent azulene-based fluoride sensor that also exhibits a colorimetric response (green to violet) in DMSO. To our knowledge, there are no published reports of azulene-boronate colorimetric fluoride indicators.

In a global development context, it would be highly desirable to develop a detection system that could be used in the field by a non-expert user (e.g. a village resident) and which requires no electrical supply or specialist equipment. A colorimetric system could potentially fulfil these requirements: a colour change that was sufficiently rapid and unambiguous, that could be discerned with the naked eye, and which did not require the user to be literate would be a useful tool for village communities to make an initial semi-quantitative assessment of drinking water safety as regards fluoride content. A crucial point is that whereas the majority of reported colorimetric fluoride detectors have been evaluated in organic solvents, clearly such a system will only be of use in a drinking water safety context if it can detect fluoride in a sample of water (and preferably without the need for any organic co-solvent).

We opted to study azulene derivatives bearing a pinacolboron substituent directly attached to the azulene skeleton. Our reasons were twofold, namely ease of synthetic access and an expectation that direct conjugation of the receptor and reporter motifs would maximise readout upon fluoride binding. We expected that an azulenylboronate ester would contain an $\mathrm{sp}^{2}$ hybridized boron centre, whereas upon fluoride binding, the boron centre would necessarily adopt $\mathrm{sp}^{3}$ hybridization in the fluoroborate product, thus leading to a significant perturbation of the $\pi$ system (this concept is shown for a representative azulenylboronate ester in Scheme 1). An initial computational study evaluated the energetics of fluoride binding. The five isomeric azulene-pinacolborane candidates are depicted in Fig. 1. For the five isomers in Fig. 1, DFT calculations were performed to quantify the change in Gibbs free energy upon binding of a free fluoride anion (see Table S1 in the ESI $\dagger$ ). The binding energy varies appreciably between the positional isomers, indicative of the inhomogeneous electron distribution in the azulene $\pi$-system. Nevertheless, a negative $\Delta G$ value in each case suggests all isomers are plausible fluoride indicators.

Of the five possible isomers, we selected Az-1-Bpin and Az-2-Bpin for synthesis and evaluation in the first instance, and these may be accessed in 1 step from commercially available starting materials. A protocol reported by Sugihara, Murafuji and co-workers for the iridium catalysed $\mathrm{C}-\mathrm{H}$ borylation of azulene $\mathrm{e}^{37}$

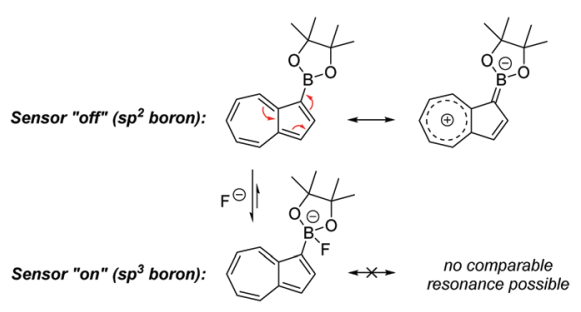

Scheme 1 Rehybridization of boron upon analyte binding.

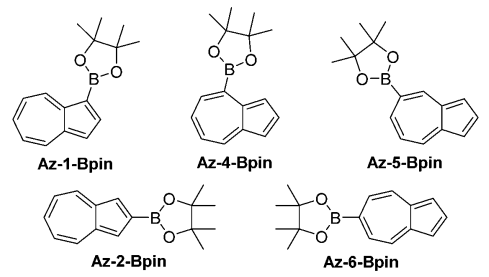

Fig. 1 Isomeric azulene-pinacolboranes evaluated computationally.

gives Az-1-Bpin and Az-2-Bpin concurrently. Az-2-Bpin is the major product (as the borylation process is sensitive to steric effects ${ }^{38}$ ), with Az-1-Bpin formed only in low yield (Scheme S1a in the ESI $\dagger$ ). However, Ingleson, Marcelli and co-workers have developed an electrophilic borylation protocol that permits an alternative selective synthesis of Az-1-Bpin as the sole isomer ${ }^{39}$ (Scheme S1b in the ESI $\dagger$ ).

Of these two regioisomers, we were able to grow crystals of Az-1-Bpin suitable for X-ray diffraction (Fig. 2). It can be seen that the dioxaborolane ring is almost coplanar with the azulene rings - the dihedral angle $\mathrm{C} 2-\mathrm{C} 1-\mathrm{B}-\mathrm{O}$ is $6.2(4)^{\circ}$, with the trigonal planar $\mathrm{sp}^{2}$ hybridized boron clearly visible.

Next, evaluation of Az-1-Bpin and Az-2-Bpin as colorimetric fluoride indicators was undertaken. Preliminary selectivity tests were performed in THF (Fig. 3). Thus, halide anions were added as their corresponding tetra- $n$-butyl ammonium (TBA) salts. Absorbance spectra were acquired after 30 seconds and 30 minutes. In each instance, the concentration of indicator was $100 \mu \mathrm{M}$ and the concentration of analyte was $180 \mu \mathrm{M}$; in the case of TBAF, this
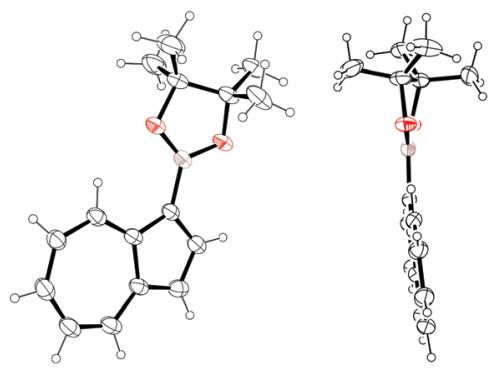

Fig. 2 Two ORTEP representations of the X-ray structure of Az-1-Bpin. Ellipsoids are shown at $50 \%$ probability.

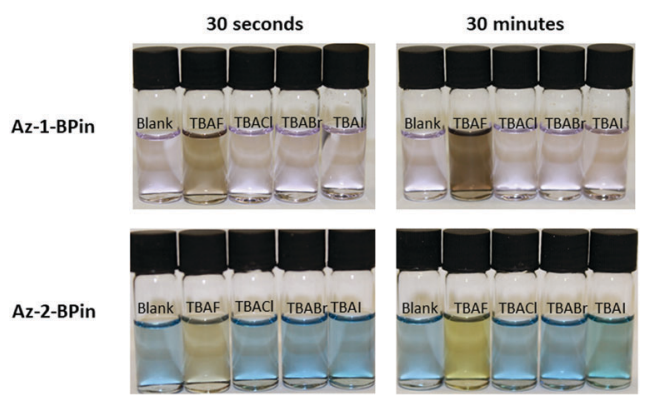

Fig. 3 Photograph of test solutions of Az-1-Bpin and Az-2-Bpin upon exposure to tetra- $n$-butylammonium halides in THF. In each solution, [analyte] $=180 \mu \mathrm{M}$, which for TBAF corresponds to $3.4 \mathrm{mg} \mathrm{L}^{-1}$ of fluoride anion. (For absorption spectra, see Fig. S1 in the ESI $\dagger$ ). 
corresponds to a loading of fluoride anion of $3.4 \mathrm{mg} \mathrm{L}^{-1}$, i.e. higher than the WHO limit of $1.5 \mathrm{mg} \mathrm{L}^{-1}$, yet lower than groundwater concentrations in many regions. For both indicators, a pronounced and selective colorimetric response to fluoride is observed - pink to brown with Az-1-Bpin, and blue to yellow with Az-2-Bpin. No other halides induced any visible change. The colour changes are most pronounced after 30 minutes, but are also clearly visible after 30 seconds.

Az-1-Bpin and Az-2-Bpin were then titrated against TBAF in THF (Fig. S2 in the ESI $\dagger$ ). An isosbestic point is observable in the UV region for Az-1-Bpin, indicating that upon introduction of fluoride, Az-1-Bpin forms the corresponding fluoroborate directly, and not through the intermediacy of any other species. A Job's plot for Az-1-Bpin showed a 1:1 stoichiometry of binding (Fig. S3 in the ESI $\dagger$ ). In contrast, the titration of Az-2-Bpin did not exhibit any isosbestic points. This may be due to the Az-2-Bpin fluoroborate reacting further (e.g. hydrolysis, formation of trifluoroborate, etc.)

Azulene-boronate ester conjugates are viable colorimetric fluoride indicators, displaying favourable analyte selectivity and speed of response. Nevertheless, as noted previously, to be useful in a drinking water context, a colorimetric fluoride indicator must detect fluoride in water itself. To this end, we attempted to determine if Az-1-Bpin or Az-2-Bpin exhibited a response to fluoride in water. However, no colour change was seen for either indicator, neither in pure water, nor in water/ organic solvent mixtures such as $\mathrm{THF} /$ water and $\mathrm{MeCN} /$ water.

Challenges for colorimetric fluoride detection in water include poor solubility of organic indicator molecules, as well as the extensive solvation shell around fluoride. Various strategies have been reported to address these issues, ${ }^{40-46}$ including the use of cetyltrimethylammonium bromide (CTAB) as a surfactant $^{47-54}$ (although CTAB can be deleterious for fluoride sensing in some contexts ${ }^{55}$ ). The surfactant should solvate the indicator within the hydrophobic micelle interior and such a concept is precedented in reports on the use of surfactant systems to enhance the nucleophilicity of fluoride for synthetic chemistry. ${ }^{56,57}$ The addition of an appropriate counter-cation to enable transfer of fluoride into the micelle is also required, as previously described; ${ }^{58}$ we employed tetra- $n$-butylammonium hydrogensulfate (TBAS) in this role.

Aqueous solutions of Az-1-Bpin, CTAB, and TBAS were treated with solutions of sodium fluoride, and as hoped, a colorimetric response was observed. Then, the interactions of Az-1-Bpin with the micelles were studied in detail by smallangle X-Ray scattering. The data were analysed using the Guinier approximation in order to obtain information about the influence of the indicator on the micelle morphology. This technique allows calculation of the radius of gyration $\left(R_{\mathrm{g}}\right)$ of the CTAB micelles with and without the indicator (see ESI $\dagger$ ).

Our results (Fig. 4) clearly show that the presence of Az-1Bpin alters the micellar environment. The change in the scattering cross section at low- $q$ (from a flat signal for the system without indicator to an increased negative slope when indicator is added) also suggests that the aggregates undergo a morphology transition when the indicator is added to the micelles.
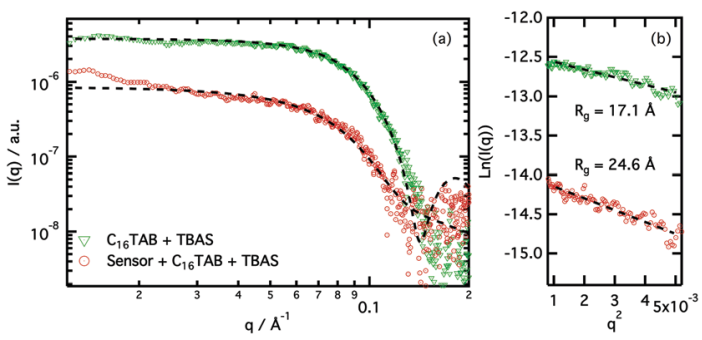

Fig. 4 (a) Small-angle $X$-ray scattering data and best fits (black-dashed lines) to the model described in the text of CTAB/TBAS before (green) and after (red) the incorporation of the Az-1-Bpin indicator (b) Guinier plot.

Guinier analysis shows that the adsorption of indicator molecules to the micelles modifies the $R_{\mathrm{g}}$ of the aggregates, from $17.1 \pm 1.2 \AA$ to $24.6 \pm 2.5 \AA$ A. More detailed morphological information on the system can be obtained through data fitting using a mathematical model (see ESI $\dagger$ for details). The fits show that the presence of Az-1-Bpin modifies the morphology of the aggregates, promoting the formation of more elongated aggregates in the presence of indicator. The rotational radius of the micelle (minor axis) is satisfactorily fitted to $29 \pm 2 \AA$ for both systems. However the aspect ratio of the micelle ( $\mathrm{AR}=$ major axis/minor axis) changes from 1.13 to 2.04 for the systems without and with the indicator, respectively. This is not surprising given the amphiphilic character of the indicator, wherein the aromatic reporter region may show comparatively hydrophobic character and migrate to the micelle core, whereas the binding region may prefer to sit at the micelle interface. ${ }^{59}$ This scenario would explain a change in the packing parameter and produce the morphology transition observed.

The water/CTAB/TBAS/Az-1-Bpin system was then evaluated with a wider variety of anions in order to determine the selectivity of the indicator (Fig. 5 and Fig. S4, ESI $\dagger$ ). The colours observable to the naked eye are somewhat different to those observed in THF (Fig. 3), especially for positive responses. This may be due to solvatochromic effects, to which azulenes are known to be prone. ${ }^{60}$ Nevertheless, a colour change is still discernible with fluoride and not with any of the other anions. Finally, Az-1-Bpin was evaluated with real samples of drinking water (Fig. S5 in the ESI $\dagger$ ), and performed comparably.

In conclusion, we have described the first colorimetric indicators for fluoride comprising a boronate ester receptor and an azulenyl reporter. Az-1-Bpin is selective for fluoride over other halides and anions commonly found in drinking water; it provides a colorimetric readout both in organic solvent and in aqueous solution (upon addition of surfactants).

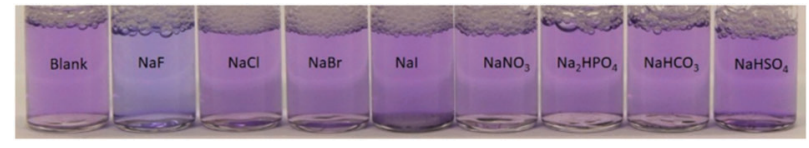

Fig. 5 Selectivity tests were performed on Az-1-BPin. Each vial contains $0.2 \mathrm{~mL}$ of indicator $(5 \mathrm{mM})$ in water/CTAB $(0.01 \mathrm{M}), 0.6 \mathrm{~mL}$ of sodium salt in DI water (7 mM) and $0.2 \mathrm{~mL}$ TBAS (0.1 M) in water. Photo taken $30 \mathrm{~min}$ after mixing. 
Current investigations on the micellar system are focused on improving the sensitivity and rendering the colour change more pronounced.

We are grateful for PhD funding to C. M. L.-A. from the EU Horizon 2020 research and innovation programme under grant agreement H2020-MSCA-CO-FUND, \#665992. The Centre for Sustainable Chemical Technologies is supported by EPSRC under grant EP/L016354/1. We also thank the European Spallation Source and University of Bath Alumni Fund for funding to A. S.-F., and EPSRC for a PhD studentship to A. C. S. This research made use of the Balena High Performance Computing (HPC) Service at the University of Bath.

\section{Conflicts of interest}

There are no conflicts to declare.

\section{Notes and references}

1 R. Liteplo, R. Gomes, P. Howe and H. Malcom, Environmental health criteria 227, World Health Organization, Geneva, 2002.

2 D. L. Ozsvath, Rev. Environ. Sci. Bio/Technol., 2009, 8, 59.

3 R. Carpenter, Geochim. Cosmochim. Acta, 1969, 33, 1153.

4 Fluoride in drinking-water, Background document for development of WHO Guidelines for drinking-water quality, World Health Organization, 2004.

5 S. Ayoob and A. K. Gupta, Crit. Rev. Environ. Sci. Technol., 2006, 36, 433. 6 R. H. Selwitz, A. I. Ismail and N. B. Pitts, Lancet, 2007, 369, 51.

7 A. Ghosh, K. Mukherjee, S. K. Ghosh and B. Saha, Res. Chem. Intermed., 2013, 39, 2881.

8 S. A. Atkinson, S. A. Abrams and L. H. Allen, Standing committee on the scientific evaluation of dietary reference intakes, food and nutrition board, Institute of Medicine, 1997.

9 M. Amini, K. Mueller, K. C. Abbaspour, T. Rosenberg, M. Afyuni, K. N. Møller, M. Sarr and C. A. Johnson, Environ. Sci. Technol., 2008, 42, 3662 .

10 Guidelines for drinking-water quality, World Health Organization, Geneva, 2011.

11 United States Environmental Protection Agency, National Primary Drinking Water Regulations, https://www.epa.gov/ground-waterand-drinking-water/national-primary-drinking-water-regulations.

12 A. Dhillon, M. Nair and D. Kumar, Anal. Methods, 2016, 8, 5338.

13 E. W. Rice and L. Bridgewater, Standard Methods for the Examination of Water and Wastewater, American Public Health Association, 2012.

14 S. Levin, S. Krishnan, S. Rajkumar, N. Halery and P. Balkunde, Sci. Total Environ., 2016, 551-552, 101.

15 M. Cametti and K. Rissanen, Chem. Commun., 2009, 2809.

16 C. R. Wade, A. E. J. Broomsgrove, S. Aldridge and F. P. Gabbaï, Chem. Rev., 2010, 110, 3958.

17 H. N. Kim, Z. Guo, W. Zhu, J. Yoon and H. Tian, Chem. Soc. Rev., $2011,40,79$

18 Y. Zhou, J. F. Zhang and J. Yoon, Chem. Rev., 2014, 114, 5511.

19 E. Quinlan, S. E. Matthews and T. Gunnlaugsson, J. Org. Chem., 2007, 72, 7497.

20 S. Rochat and K. Severin, Chem. Commun., 2011, 47, 4391.

21 T. Liu, A. Nonat, M. Beyler, M. Regueiro-Figueroa, K. N. Nono, O. Jeannin, F. Camerel, F. Debaene, S. Cianférani-Sanglier, R. Tripier, C. PlatasIglesias and L. J. Charbonnière, Angew. Chem., Int. Ed., 2014, 53, 7259.

22 S. J. Butler, Chem. Commun., 2015, 51, 10879.

23 For an example, see: T. Nishimura, S.-Y. Xu, Y.-B. Jiang, J. S. Fossey, K. Sakurai, S. D. Bull and T. D. James, Chem. Commun., 2013, 49, 478.
24 K. Abou-Hadeed and H.-J. Hansen, Sci. Synth., 2009, 45b, 1043.

25 P. Cowper, Y. Jin, M. D. Turton, G. Kociok-Köhn and S. E. Lewis, Angew. Chem., Int. Ed., 2016, 55, 2564.

26 R. S. H. Liu and A. E. Asato, J. Photochem. Photobiol., C, 2003, 4, 179. 27 D. Lichosyt, P. Dydio and J. Jurczak, Chem. - Eur. J., 2016, 22, 17673.

28 T. Zielinski, M. Kedziorek and J. Jurczak, Chem. - Eur. J., 2008, 14, 838.

29 T. Zielinski, M. Kedziorek and J. Jurczak, Tetrahedron Lett., 2005, 46, 6231.

30 S. H. Kim, Y. A. Son, J. S. Bae and D. H. Lee, Korean Pat., KR2014078467, June 25, 2014.

31 J. T. Ippoliti and K. E. Olson, US Pat., US20080286812, November 20, 2008.

32 S. Wakabayashi, M. Uchida, R. Tanaka, Y. Habata and M. Shimizu, Asian J. Org. Chem., 2013, 2, 786.

33 S. Wakabayashi, Y. Kato, K. Mochizuki, R. Suzuki, M. Matsumoto, Y. Sugihara and M. Shimizu, J. Org. Chem., 2007, 72, 744.

34 S. Wakabayashi, R. Uriu, T. Asakura, C. Akamatsu and Y. Sugihara, Heterocycles, 2008, 75, 383.

35 S. Wakabayashi, R. Yamaoka, E. Matsumoto, M. Nishiguchi, M. Ishiura, M. Tsuji and M. Shimizu, Heterocycles, 2012, 85, 2251.

36 H. Salman, Y. Abraham, S. Tal, S. Meltzman, M. Kapon, N. Tessler, S. Speiser and Y. Eichen, Eur. J. Org. Chem., 2005, 2207.

37 K. Kurotobi, M. Miyauchi, K. Takakura, T. Murafuji and Y. Sugihara, Eur. J. Org. Chem., 2003, 3663.

38 M. Fujinaga, T. Murafuji, K. Kurotobi and Y. Sugihara, Tetrahedron, 2009, 65, 7115.

39 V. Bagutski, A. del Grosso, J. A. Carrillo, I. A. Cade, M. D. Helm, J. R. Lawson, P. J. Singleton, S. A. Solomon, T. Marcelli and M. J. Ingleson, J. Am. Chem. Soc., 2013, 135, 474.

40 M. S. Baker and S. T. Phillips, Org. Biomol. Chem., 2012, 10, 3595.

41 B. Ke, W. Chen, N. Ni, Y. Cheng, C. Dai, H. Dinh and B. A. Wang, Chem. Commun., 2013, 49, 2494.

42 K. Kanagaraj and K. Pitchumani, Chem. - Asian J., 2014, 9, 146.

43 Q. Tang, H.-M. Nie, C.-B. Gong, H.-D. Liu and K. A. Xiao, RSC Adv., 2015, 5, 3888.

44 Y. Zheng, Y. Duan, K. Ji, R.-L. Wang and B. Wang, RSC Adv., 2016, 6, 25242 .

45 M. Yu, J. Xu, C. Peng, Z. Li, C. Liu and L. Wei, Tetrahedron, 2016, 72, 273.

46 A. Kumar, M. Bhatt, G. Vyas, S. Bhatt and P. Paul, ACS Appl. Mater. Interfaces, 2017, 9, 17359.

47 R. Hu, J. Feng, D. Hu, S. Wang, S. Li, Y. Li and G. Yang, Angew. Chem., Int. Ed., 2010, 49, 4915.

48 G. Wei, J. Yin, X. Ma, S. Yu, D. Wei and Y. Du, Anal. Chim. Acta, 2011, 703, 219.

49 L. K. Calderon-Ortiz, E. Taeuscher, E. Leite Bastos, H. Goerls, D. Weiss and R. Beckert, Eur. J. Org. Chem., 2012, 2535.

50 S. Elsayed, A. Agostini, L. E. Santos-Figueroa, R. Martinez-Manez and F. Sancenon, ChemistryOpen, 2013, 2, 58.

51 B. Qiu, T.-J. Yu, J.-P. Chen, Y. Zeng and Y. Li, Imaging Sci. Photochem., 2013, 31, 383, DOI: 10.7517/j.issn.1674-0475.2013.05.383.

52 A. Roy, T. Saha and P. Talukdar, Tetrahedron Lett., 2015, 56, 4975.

53 H. Wang, P.-H. Fan, B. Tong, Y.-P. Dong, X.-M. Ou and F. Li, Adv. Funct. Mater., 2015, 25, 1506.

54 B. Qiu, Y. Zeng, L. Cao, R. Hu, X. Zhang, T. Yu, J. Chen, G. Yang and Y. Li, RSC Adv., 2016, 6, 49158.

55 X. Wu, X.-X. Chen, B.-N. Song, Y.-J. Huang, W.-J. Ouyang, Z. Li, T. D. James and Y.-B. Jiang, Chem. Commun., 2014, 50, 13987.

56 C. A. Bunton and L. B. Robinson, J. Org. Chem., 1969, 34, 773.

57 C. A. Bunton, J. Mol. Liq., 1997, 72, 231.

58 For example, see the ESI in the following reference: A. Wallabregue, D. Moreau, P. Sherin, P. Moneva Lorente, Z. Jarolímová, E. Bakker, E. Vauthey, J. Gruenberg and J. Lacour, J. Am. Chem. Soc., 2016, $138,1752$.

59 C. A. Dreiss, Soft Matter, 2007, 3, 956.

60 A. Blice-Baum, A. Van Dyke, I. Sigmon, E. A. Salter, A. Wierzbicki, Y. Pocker and G. T. Spyridis, Int. J. Quantum Chem., 2006, 106, 2331. 